\title{
Bowman-Birk protease inhibitor from soybeans enhances cisplatin-induced cytotoxicity in human mesothelioma cells
}

\author{
KOREHITO KASHIWAGI ${ }^{1,2,3}$, NANTIGA VIRGONA ${ }^{3}$, JIN YAMADA ${ }^{1}$, AYAMI SATO ${ }^{1}$, \\ MASAKO OTA $^{1}$, TAKUYA YAZAWA ${ }^{2}$ and TOMOHIRO YANO ${ }^{1,3}$ \\ ${ }^{1}$ Faculty of Life Sciences, Toyo University, Itakura, Gunma 374-0192; ${ }^{2}$ Department of Pathology, Yokohama City University, \\ Graduate School of Medicine, Kanazawa, Yokohama 236-0004; ${ }^{3}$ Project for Complementary Medicine, \\ National Institute of Health and Nutrition, Shinjuku, Tokyo 162-8636, Japan
}

Received March 8, 2011; Accepted April 11, 2011

DOI: $10.3892 /$ etm.2011.270

\begin{abstract}
Malignant mesothelioma (MM) is an aggressive cancer with no effective treatment options. Enforced expression of the gap junction (GJ) component connexin 43 (Cx43) increases the sensitivity of MM cells to cisplatin. BowmanBirk protease inhibitor (BBI) induces the restoration of $\mathrm{Cx} 43$ in several types of tumor cells. In this study, we examined the capability of BBI to enhance the cytotoxic effect of cisplatin in MM cells via the induction of Cx43. Human MM H28 cells were used. Cell viability was evaluated by a WST-1 assay and proteasomal activity was determined by fluorometric analysis. Protein and mRNA levels were determined by immunoblot analysis and real-time RT-PCR, respectively. GJ function mediated by $\mathrm{Cx} 43$ was evaluated using the scrapeloading method. BBI effectively inhibited H28 cell growth in a dose-dependent manner (200-400 $\mu \mathrm{g} / \mathrm{ml})$. In parallel with the growth inhibition, $\mathrm{Cx} 43$ levels (mRNA and protein) and GJ function were elevated by BBI treatment. Knockdown of BBI-induced $\mathrm{Cx} 43$ by an antisense nucleotide treatment almost cancelled the growth inhibition. BBI enhanced cisplatin-induced cytotoxicity in $\mathrm{H} 28$ cells, and down-regulation of $\mathrm{Cx} 43$ by the antisense nucleotide treatment abrogated the enhancing effect of BBI. The induction of $\mathrm{Cx} 43$ by BBI contributed to Src inactivation and subsequent induction of Bax. Furthermore, an Src inhibitor (SU6656) also enhanced cisplatin-induced cytotoxicity in $\mathrm{H} 28$ cells. These results suggest that BBI improves the cytotoxic efficacy of cisplatin in $\mathrm{H} 28$ cells via the inhibition of Src signaling.
\end{abstract}

\section{Introduction}

Malignant mesothelioma (MM) of the serosal membranes of body cavities is a particularly aggressive cancer characterized by rapid progression, late metastases and poor prognosis (1).

Correspondence to: Dr Tomohiro Yano, Faculty of Life Sciences, Toyo University, 1-1-1 Izumino, Itakura, Oura, Gunma 374-0192, Japan E-mail: ya.tomohiro@gmail.com

Key words: chemoresistance, cisplatin, Bowman-Birk protease inhibitor, mesothelioma, Src
Although surgery, radiotherapy, chemotherapy and/or their combinations have been used as therapeutic modalities, median patient survival is 8-18 months (2). Cisplatin has been used in clinical MM therapy, and its chemotherapeutic effect as a single agent as well as in combination with other chemotherapeutic agents has been previously examined $(3,4)$. However, most patients relapsed within 1 year after starting treatment. Therefore, new therapeutic approaches are required for MM patients.

Among the different types of cell-cell interactions in mammalian cells, gap junctional intercellular communication (GJIC) is considered to be the only route allowing free direct transfer of ions and hydrophilic molecules of up to $1,000-1,500 \mathrm{Da}$ in size between cells, thereby maintaining electrical and metabolic cell homeostasis (5). The gap junction is made up of juxtaposed transmembrane hemichannels (connexons) provided by adjacent cells, and each connexon consists of six individual transmembrane proteins called connexin $(\mathrm{Cx})(6)$. In general, it is well known that the $\mathrm{Cx}$ gene acts as a tumor-suppressor gene by maintaining homeostatic control in multicellular organisms via GJIC. Moreover, many transfection studies using $\mathrm{Cx}$ cDNAs revealed that $\mathrm{Cx}$ is a tumor suppressor in cells originating from tissues in which they are normally expressed (7). In line with this, we recently reported that $\mathrm{Cx} 43$ abrogates various malignant phenotypes in MM cells, such as chemoresistance (8).

Protease inhibitors are a class of well-established cancer chemopreventive agents due to their strong anticarcinogenic activity in vivo and in vitro in cancer model systems (9). The most predominant protease inhibitor in soybeans is the Bowman-Birk inhibitor (BBI) (9). BBI, a 71-amino acid protein $(8 \mathrm{kDa})$ and a serine protease inhibitor with both trypsin and chymotrypsin inhibitory activities, was found to be a valid suppressor of carcinogenesis in a human phase IIa clinical trial (10). Although BBI has a broad spectrum of cancer-protective activities (10-12), knowledge of the exact mechanism(s) by which BBI exerts its anticarcinogenic effects remains limited. In our previous studies, we demonstrated that the induction of $\mathrm{Cx} 43$ by BBI contributes to the negative growth control of tumor cells in vivo as well as in vitro $(13,14)$. Overall, it appears that BBI improves chemoresistance in MM cells via the induction of $\mathrm{Cx} 43$. 
In this context, we evaluated whether BBI enhances cisplatin-induced cytotoxicity in MM cells. The H28 human MM cell line was chosen to evaluate the inhibitory effect of BBI on MM cell growth, due to its resistance to cisplatin, a representative agent used to clinically treat MM.

\section{Materials and methods}

Chemicals. All culture chemicals and BBI were purchased from Gibco BRL (Tokyo, Japan) and Sigma (St. Louis, MO, USA), unless otherwise indicated. SU6656 (an Src inhibitor) was purchased from Calbiochem-Novabiochem (La Jolla, CA, USA). Anti-Cx43 antibody was from Zymed Laboratories (San Francisco, CA, USA). Other antibodies were purchased from Wako Pure Chemicals (Osaka, Japan) and BD Transduction Laboratories (Lexington, KY, USA).

Cell culture and treatment. Human mesothelioma H28 cell line was supplied by ATCC, and the cells were cultured in RPMI-1640 supplemented with $10 \%$ fetal bovine serum (FBS), 0.01 M HEPES buffer solution, $1 \mathrm{mM}$ sodium pyruvate and $4.5 \mathrm{~g} / 1$ glucose in a $5 \% \mathrm{CO}_{2}$ atmosphere at $37^{\circ} \mathrm{C}$ in a humidified incubator. BBI was dissolved in saline, and the cells were treated with BBI (200 and $400 \mu \mathrm{g} / \mathrm{ml})$ or vehicle (saline) for indicated periods. Treatment conditions involving the other agents were performed as described in each figure legend.

Cell growth assay. Cells $\left(2 \times 10^{4}\right)$ were seeded on a 96-well culture plate with the culture medium, and after overnight culture, the cells were treated with each agent. Following this, cell growth was determined with a cell proliferation assay kit using WST-1 reagent (Roche Japan, Tokyo, Japan).

Apoptosis analysis. Cells were trypsinization, washed with PBS, resuspended in $70 \%$ ethanol and maintained at $4^{\circ} \mathrm{C}$ for at least $30 \mathrm{~min}$. Before analysis, the cells were washed again with PBS and resuspended and incubated for $30 \mathrm{~min}$ in PBS containing $0.05 \mathrm{mg} / \mathrm{ml}$ propidium iodide, $1 \mathrm{mM}$ EDTA, $0.1 \%$ Triton X-100 and $1 \mathrm{mg} / \mathrm{ml}$ RNase A. The suspension was then passed through a nylon mesh filter and analyzed on a Becton Dickinson FACScan.

Measurement of GJIC. For measurement of GJIC, we used a scrape-loading/dye transfer method with some modification (15). H28 cells on 35-mm dishes were rinsed several times with PBS. The center of the dish was scraped by a surgical blade, and $2 \mathrm{ml}$ of $0.05 \%$ Lucifer yellow $\mathrm{CH}$ (LY) in PBS was added to the dishes after scraping. LY is a small molecule (457 Da) that freely moves through gap junctions from loaded cells to neighboring ones. Five minutes after the dye treatment, the cells were rinsed several times with PBS to remove excess dye. The intensity of LY transfer was observed with an Olympus inverse microscope equipped with appropriate filters (Olympus, Tokyo, Japan), five points were photographed per dish and the cell layers into which LY had spread were counted.

Cx43 antisense phosphorothioate oligodeoxynucleotide treatment. Cx43 antisense phosphorothioate oligodeoxynucleotide
(AS-ODN) (5'-CTCCAGTCACCCATGTTG-3') was purchased from Sigma-Genosys (Hokkaido, Japan). The sequence encompassed the start codon of rat $\mathrm{Cx} 43 \mathrm{mRNA}$. As a control for the non-specific effects of oligonucleotide treatment, the corresponding sense phosphorothioate ODN (S-ODN) (5'-CAACATGGGTGACTGGAG-3') was also obtained from Sigma Genosys. The cells were exposed to $2 \mu \mathrm{M}$ AS-ODN or S-ODN and added to the culture medium at 2-day intervals. Under these conditions, the expression of Cx43 protein was almost diminished in the cells treated with AS-ODN, while S-ODN treatment at the equivalent dose did not affect the protein level.

Isolation of total RNA and real-time PCR. Total RNA was isolated by using the SV Total RNA isolation system (Promega, Madison, WI, USA), and cDNA was synthesized as previously described (8). Real-time PCR was performed by using ABI Prism 7000 sequence detection system (Applied Biosystems Japan Ltd., Tokyo, Japan) and SYBR Premix Ex Taq $^{\text {TM }}$ (Takara Bio Inc., Shiga, Japan) according to the manufacturer's instructions. The primers used were as follows: glyceraldehyde-3-phosphate dehydrogenase (GAPDH), accession no. (BC023632), sense (nucleotides 737-756), antisense (nucleotides 916-897); Cx43, accession no. (NM_000165), sense (nucleotides 257-276) and antisense (nucleotides 433-414).

Immunoblot analysis. Immunoblotting was performed as previously described (14). Briefly, the cell lysate was prepared in cell lysis/extraction reagent (Sigma), and $10 \mu \mathrm{g}$ total protein extract from each sample was loaded onto $8 \%$ SDS-polyacrylamide gel. After electrophoresis, proteins were transferred to nitrocellulose membranes. The blots were incubated with each antibody. Each immunoreactive band was detected using the ECL system (Amersham) and a cooled CCD camera-linked Cool Saver system (Atto, Osaka Japan). Molecular sizing was carried out using Rainbow MW marker (Amersham). Protein concentrations were determined using the DC protein assay system (Bio-Rad, Hercules, CA, USA).

Assay for proteasome activity. H28 cells were seeded on a 12 -well plate $\left(5 \times 10^{4}\right.$ cells/well $)$ overnight. These cells were then treated with the specific concentration of BBI for 24 or $72 \mathrm{~h}$, followed by the addition of $20 \mu \mathrm{M}$ of flurogenic peptide substrate Suc-Leu-Leu-Val-Tyr-AMC (for chymotrypsinlike activity) at $37^{\circ} \mathrm{C}$ for $2 \mathrm{~h}$. Afterwards, $100 \mu \mathrm{l}$ of the cell medium was collected and diluted with $1 \mathrm{X}$ PBS to $400 \mu \mathrm{l}$. Measurement of free AMC groups was performed as previously described (16).

Statistical analysis. Data were analyzed by one-way ANOVA followed by the Student's t-test, Dunnett's multiple-range test or Tukey-Kramer test. $\mathrm{P} \leq 0.05$ was considered statistically significant.

\section{Results}

Effect of BBI on cell growth, proteasomal activity and C43 expression in H28 cells. To evaluate the effect of BBI on cell growth, proteasomal activity and Cx43 expression level, 


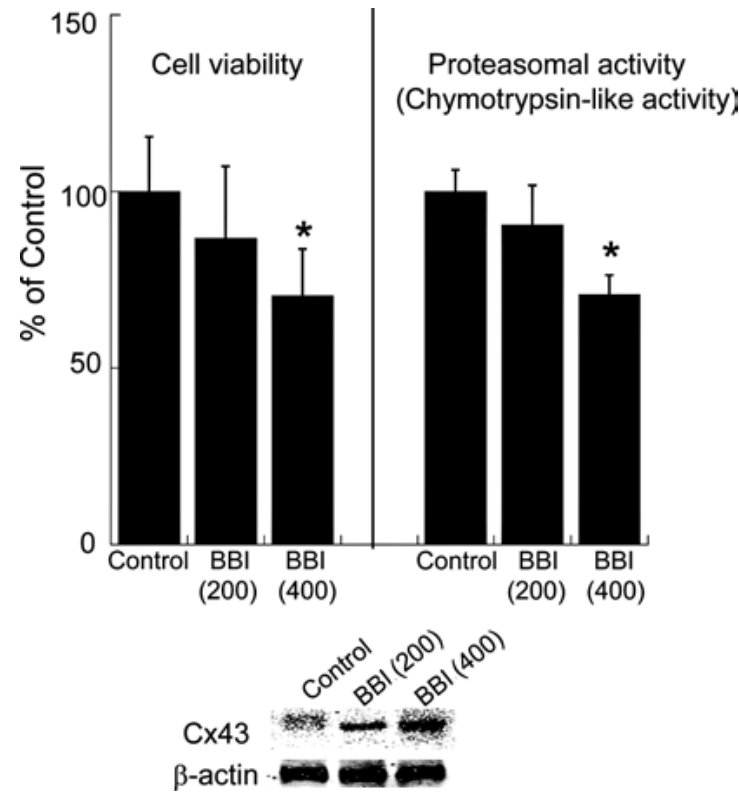

Figure 1. Effects of BBI on cell viability, proteasomal activity of chymotrypsin-like activity and the $\mathrm{Cx} 43$ protein level of $\mathrm{H} 28$ cells. The cells were treated with BBI $(200 \mu \mathrm{g} / \mathrm{ml})$ and BBI $(400 \mu \mathrm{g} / \mathrm{ml})$ for 5 days (cell viability) and 3 days (proteasomal activity and $\mathrm{Cx} 43$ protein level), respectively. Each value is the mean from five determinants, and vertical lines indicate SD (cell viability and proteasomal activity). "Significant difference from control. The level of $\mathrm{Cx} 43$ protein was determined by immunoblot analysis, and $\beta$-actin was used to confirm equal loading between each group. This result is representative of two independent experiments. BBI (200), BBI $200 \mu \mathrm{g} / \mathrm{ml}$; BBI (400), BBI $400 \mu \mathrm{g} / \mathrm{ml}$.

dose-dependent changes in cell viability, chymotrypsin-like activity involved in the proteasome and the protein level of Cx43 after BBI exposure for each time period were examined. Since the elevation of Cx43 by BBI partly depends on the inhibition of chymotrypsin-like activity in the proteasome (14), we assessed the activity in the proteasome to confirm the effect of BBI. As shown in Fig. 1, BBI decreased cell viability and suppressed proteasomal chymotrypsin-like activity in a dose-dependent manner, whereas the expression level of Cx43 protein was dose-dependently elevated by BBI treatment. Next, the effect of BBI on the Cx43 mRNA level and GJIC in H28 cells was investigated (Fig. 2). BBI treatment up-regulated the Cx43 mRNA level and in parallel caused the restoration of GJIC estimated by the scrape-loading assay. Finally, to confirm the contribution of the BBI-mediated elevation of $\mathrm{Cx} 43$ to the negative growth effect in $\mathrm{H} 28$ cells, we ascertained whether the knockdown of $\mathrm{Cx} 43$ by AS-ODN abrogates the BBI-dependent negative growth effect in H28 cells. As shown in Fig. 3, Cx43 AS-ODN almost completely abolished BBI-mediated growth inhibition in $\mathrm{H} 28$ cells upon down-regulation of $\mathrm{Cx} 43$.

Combination effect of BBI and cisplatin on the growth of $H 28$ cells. We previously reported that enforced expression of the $\mathrm{Cx} 43$ gene improved the resistance of cisplatin to H28 cells (8); therefore, we investigated whether BBI enhances cisplatin-induced cytotoxicity and apoptosis in $\mathrm{H} 28$ cells. As shown in Fig. 4, cisplatin treatment $(10 \mu \mathrm{M})$ had no effect on cell viability in $\mathrm{H} 28$ cells, but the combination of BBI with cisplatin significantly decreased the cell viability compared
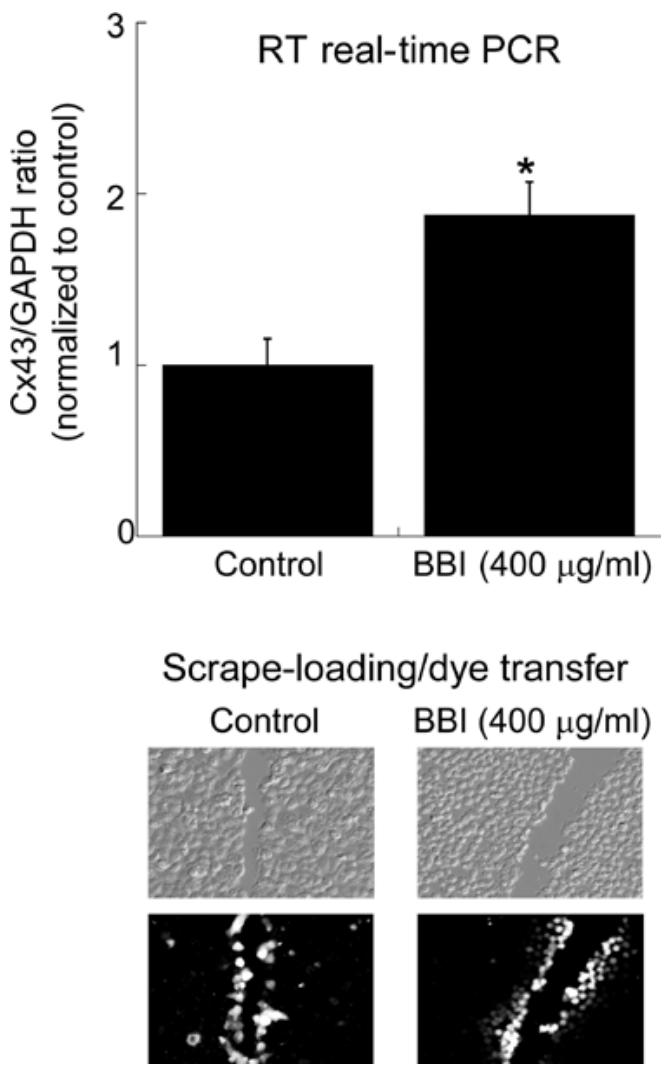

Figure 2. Restoration of Cx43 mRNA expression and GJIC in H28 cells treated with BBI. The cells were treated with BBI $(400 \mu \mathrm{g} / \mathrm{ml})$ for 3 days. The level of Cx43 mRNA was determined by real-time RT-PCR. Each value is the mean from five determinants, and vertical lines indicate SD. *Significant difference from control. GJIC was estimated by scrape-loading/ dye transfer method. Original magnification, $\mathrm{x} 200$. This result is representative of two independent experiments.

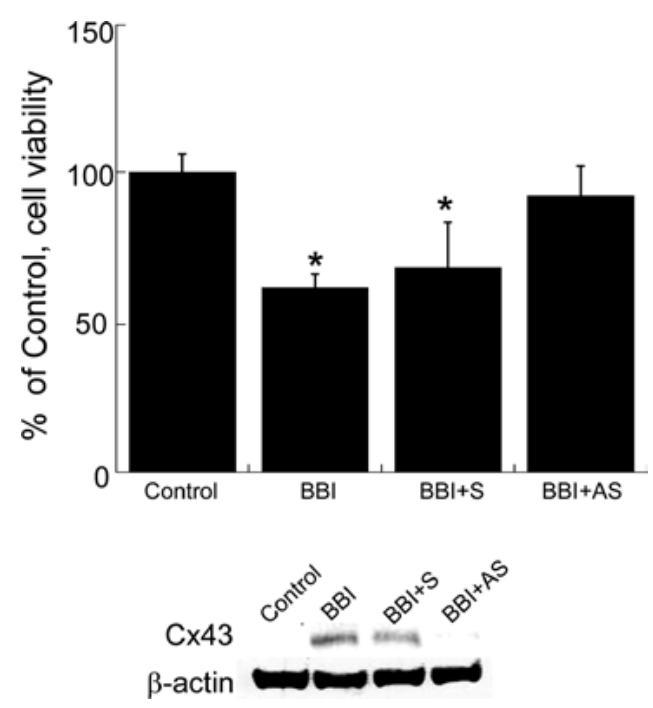

Figure 3. Change in cell viability in BBI-treated $\mathrm{H} 28$ cells upon the downregulation of $\mathrm{Cx} 43$ by AS-ODN. The cells were treated with BBI (400 $\mu \mathrm{g} /$ $\mathrm{ml})$ and/or AS-ODN (2 M) for 5 days. After the treatment, cell viability was determined by WST-1. Each value is the mean from four determinants, and vertical lines indicate SD. *Significant difference from control. Under the same treatment condition, the Cx43 protein level in each group was estimated by immunoblot analysis. This result is representative of two independent experiments. S-ODN was used as a negative control. BBI, BBI treatment; BBI+S, BBI + sense phosphorothioate oligodeoxynucleotide treatment; $\mathrm{BBI}+\mathrm{AS}, \mathrm{BBI}+\mathrm{Cx} 43$ antisense phosphorothioate oligodeoxynucleotide treatment. 

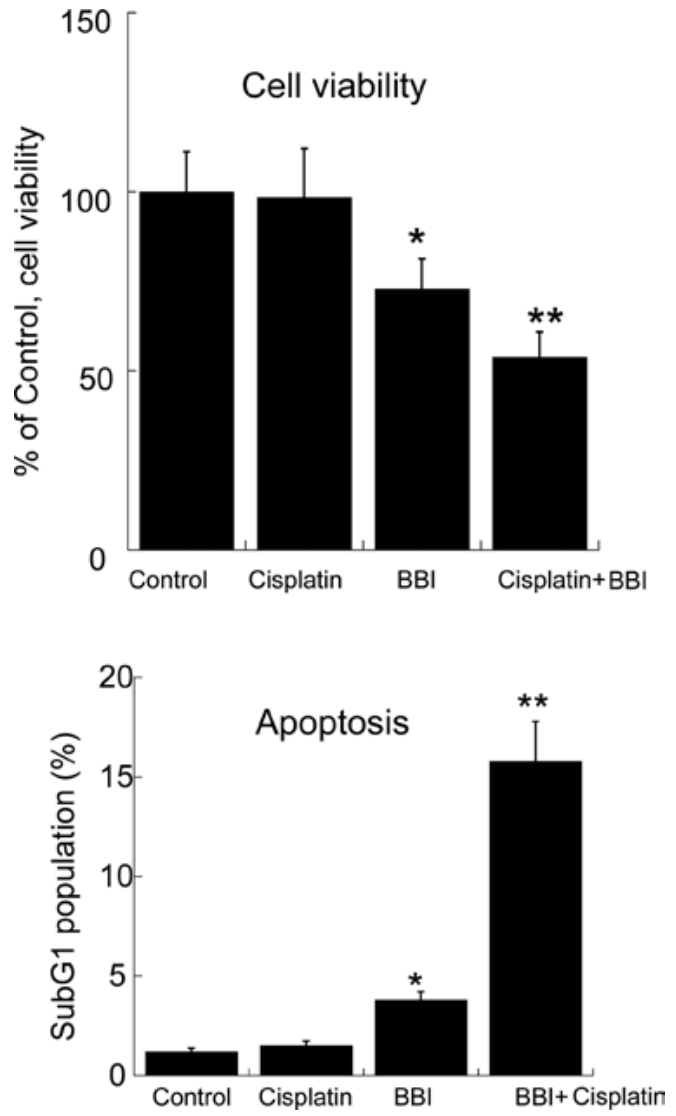

Figure 4. Effect of BBI on cell viability and apoptosis in $\mathrm{H} 28$ cells treated with cisplatin. The cells were pre-treated with BBI $(400 \mu \mathrm{g} / \mathrm{ml})$ for 3 days and subsequently co-treated with BBI and/or cisplatin $(10 \mu \mathrm{M})$ for 2 days. After the treatment, cell viability was determined by WST-1, and apoptosis was evaluated as the subG1 population. Each value is the mean from four determinants, and vertical lines indicate SD. *Significant difference from control and BBI-treated group and ${ }^{* *}$ significant difference from other three groups.

to BBI treatment alone. Also, the induction of apoptosis, estimated as the subG1 population, by the combination of BBI and cisplatin was significantly increased compared to that with BBI or cisplatin alone (Fig. 4). These results indicate that BBI abrogates the resistance to cisplatin in the cells. Furthermore, in order to ascertain whether BBI-driven restoration of $\mathrm{Cx} 43$ is closely related to the improvement of chemoresistance in $\mathrm{H} 28$ cells by $\mathrm{BBI}$, we investigated whether AS-ODN for $\mathrm{Cx} 43$ cancels BBI-enhanced cytotoxicity in $\mathrm{H} 28$ cells treated with cisplatin. As a result, the enhanced effect of BBI was almost abolished by the AS-ODN treatment (Fig. 5).

Effect of BBI on Src signaling in $\mathrm{H} 28$ cells. We previously reported that inactivation of the Src family of protein tyrosine kinases is involved in enhancing the effect of $\mathrm{Cx} 43$ on cisplatin-induced cytotoxicity in $\mathrm{H} 28$ cells (8). Therefore, we speculated that the enhancing effect of BBI on cytotoxicity is due to suppression of Src signaling via the induction of Cx43. To confirm this hypothesis, we evaluated whether BBI-enhanced Cx43 expression improves the efficacy of cisplatin to $\mathrm{H} 28$ cells via the inactivation of Src signaling. As shown in Fig. 6, BBI suppressed the activation of Src (phosphorylation on Tyr416), and knockdown of Cx43 by AS-ODN treatment almost completely cancelled this BBI-mediated

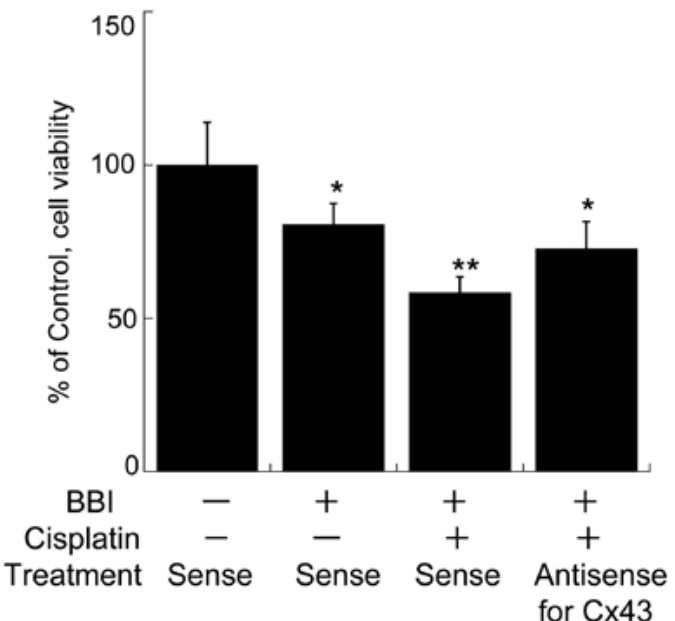

Figure 5. Effect of Cx43 AS-ODN on cisplatin-induced cytotoxicity in H28 cells treated with BBI. The cells were treated with Cx43 AS-ODN ( $2 \mu \mathrm{M})$ and BBI $(400 \mu \mathrm{g} / \mathrm{ml})$ for 3 days, and subsequently co-treated with cisplatin $(10 \mu \mathrm{M})$ for 2 days. Each value is the mean from four determinants, and vertical lines indicate SD. *Significant difference from control and ${ }^{* *}$ significant difference from other three groups.

inactivation of Src. In our previous study, Bax, a representative pro-apoptotic factor, was shown to be induced by enforced expression of $\mathrm{Cx} 43$ and to play an important role in the enhancement of cisplatin-mediated damage in $\mathrm{H} 28$ cells (8); therefore, we evaluated the contribution of Bax to the enhancing effect of BBI in $\mathrm{H} 28$ cells. In the present study, BBI treatment up-regulated Bax and, upon knockdown of $\mathrm{Cx} 43$ by AS-ODN treatment, the Bax level was almost restored to the level of that in the control (Fig. 6). Additionally, the Src inhibitor, SU6656, elevated the Bax level, indicating that inhibition of Src leads to the induction of Bax. Finally, in order to confirm that Src signaling plays a crucial role in the BBI-induced enhancement of cisplatin-mediated cytotoxicity, we evaluated the combination effect of SU6656 and cisplatin on the growth of $\mathrm{H} 28$ cells. As shown in Fig. 7, when the cells were treated with both SU6656 and cisplatin, cell viability was significantly inhibited as compared to that when the cells were treated with either SU6656 or cisplatin alone.

\section{Discussion}

Management of MM continues to be a difficult clinical issue. The relative clinical resistance to chemotherapy and other conventional treatments strongly necessitate the development of new potential therapeutic strategies for MM to establish viable MM treatment protocols. In our previous study, enforced expression of Cx43 in MM cells exhibiting resistance to cisplatin significantly reduced this resistance (8), indicating that successful restoration of $\mathrm{Cx} 43$ by a treatment agent rather than $\mathrm{Cx} 43$ gene transfection may lead to the establishment of a novel effective MM therapy. Fortunately, in regards to the restoration of $\mathrm{Cx} 43$ expression in tumor cells, we and other research groups previously found that BBI effectively caused the induction of $\mathrm{Cx} 43$ in several types of tumor cells and that this induction is closely associated with negative growth control of tumor cells $(14,17)$. Based on these reports, we speculated that BBI improves the efficacy of cisplatin in MM 
A

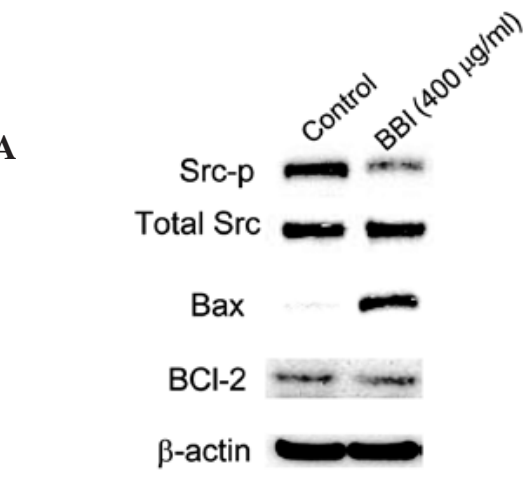

B

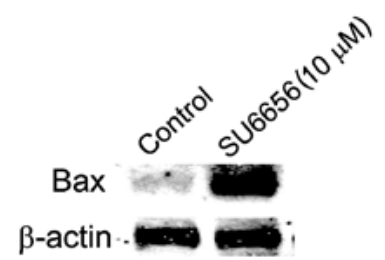

C

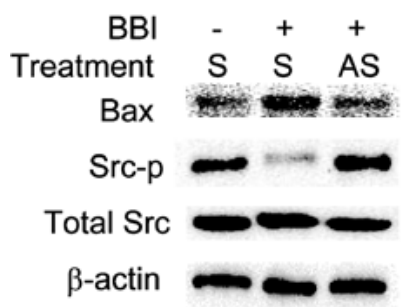

Figure 6. Contribution of $\mathrm{Cx} 43$ to the suppression of Sre signaling in $\mathrm{H} 28$ cells treated with BBI. The cells were treated with (A) BBI $(400 \mu \mathrm{g} / \mathrm{ml})$ for 3 days, (B) SU6656 $(10 \mu \mathrm{M})$ for 2 days, and (C) Cx43 AS-ODN $(2 \mu \mathrm{M})$ and BBI $(400 \mu \mathrm{g} / \mathrm{ml})$ for 3 days. After each treatment, the level of each protein was determined by immunoblot analysis. This result is representative of two independent experiments. S, sense phosphorothioate oligodeoxynucleotide treatment; AS, Cx43 antisense phosphorothioate oligodeoxynucleotide treatment.

cells via restoration of $\mathrm{Cx} 43$ expression. Thus, the present study was carried out to confirm this hypothesis.

We previously reported that the $\mathrm{Cx}$ gene (the molecule being Cx32) enhances the sensitivity of chemotherapeutic agents to renal and lung cancer cells (18). Similarly, we demonstrated that enforced $\mathrm{Cx} 43$ expression in $\mathrm{H} 28$ cells improves the effect of cisplatin against $\mathrm{H} 28$ cells (14). These reports strongly suggest that, in part, $\mathrm{Cx}$-dependent tumor suppressive effects mitigate chemoresistance in several types of tumor cells. In the present study, we showed that BBI treatment caused the restoration of $\mathrm{Cx} 43$ expression, in part, due to the suppression of $\mathrm{Cx} 43$ degradation by chymotrypsin-like activity in the proteasome, and that the restored $\mathrm{Cx} 43$ induced negative growth control in H28 cells. Finally, these events caused by BBI treatment led to an improvement in the efficacy of cisplatin. Thus, the combination of BBI and cisplatin may be a promising new therapy against cisplatin-resistant MM cells.

Through GJIC-dependent cell coupling, dying cancer cells communicate cell death signals to adjacent cells which then also die by apoptosis. These death messages which pass through the GJ to kill cells are very likely calcium ions (19).

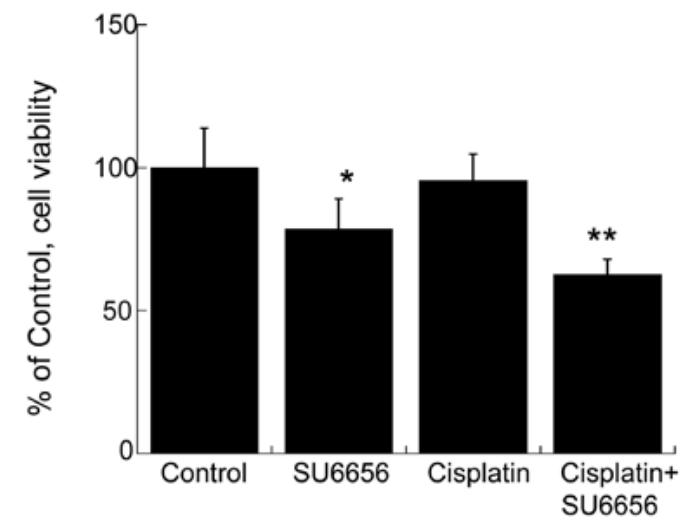

Figure 7. Enhancing effect of SU6656 on cisplatin-induced cytotoxicity in $\mathrm{H} 28$ cells. The cells were treated with SU6656 $(10 \mu \mathrm{M})$, cisplatin $(10 \mu \mathrm{M})$ and both for 2 days. After the treatment, cell viability was determined by WST-1. Each value is the mean from five determinants, and vertical lines indicate SD. *Significant difference from control and cisplatin-treated group and ${ }^{* *}$ significant difference from the other three groups.

This finding suggests that chemoresistance to anticancer agents by tumor cells is reduced due to the propagation of cell death signals from dying cells to surrounding living cells via GJ. We previously reported that inhibition of Cx-driven GJIC by a known inhibitor towards GJ functions (18-glycyrrhetinic acid) partly abrogated chemotherapeutic agent-induced cytotoxicity in cancer cells (18). However, in this study we did not find that inhibition of GJIC by the inhibitor negates the enhanced effect of BBI on cisplatin-induced cytotoxicity in $\mathrm{H} 28$ cells (data not shown), indicating that BBI-mediated restoration of GJIC does not affect cisiplatin-induced cytotoxicity. Apart from the GJIC-dependent effect of $\mathrm{Cx}$, other data showed that $\mathrm{Cx}$ affects cellular homeostatic balance independently of GJIC, and that this GJ-independent effect plays an important role in regulating abnormal growth of cancer cells $(5,20)$. Moreover, we demonstrated that the Cx32 gene acts as a tumor suppressor gene against renal cancer cells in a GJIC-independent mechanism (21). Based on these reports, we speculated that the GJIC-independent effect contributes to the potentiation of cisplatin-induced cytotoxicity in $\mathrm{H} 28$ cells.

We previously reported that one of the GJIC-independent tumor-suppressive effects in renal cancer cells depends on the inactivation of Src caused by Cx32 (21). It has been well-established that Src plays a critical role in the survival, proliferation, invasion and metastasis of solid tumors (22), and also, the activation of Src contributes to the appearance of malignant phenotypes in MM cells (23). Furthermore, Src directly and indirectly phosphorylates $\mathrm{Cx} 43$ at tyrosine and serine residues, leading to loss of $\mathrm{Cx} 43$-mediated functions (24). On the contrary, we observed that overexpression of Cx43 induces, not only dephosphorylation, but also downregulation of Src in MM cells (8). These reports suggest that these two molecules affect their respective function based on the expression level of each molecule. Thus, BBI-induced elevation of $\mathrm{Cx} 43$ may improve the toxicity of cisplatin to $\mathrm{H} 28$ cells via the inactivation of Src in a GJIC-independent manner. We confirmed that the knockdown of $\mathrm{Cx} 43$ in BBI-treated H2 8 cells by AS-ODN abrogated the enhancing effect of BBI on cisplatin-induced cytotoxicity, and that Src inhibition by a 
specific inhibitor caused the potentiation of the cytotoxicity. These observations completely support the above speculation.

In general, the existence of anti-apoptotic molecules and pro-apoptotic molecules is well known (25). Bcl-2 is a major anti-apoptotic protein which protects cells from a wide variety of apoptotic stimuli (26). By contrast, Bax is a Bcl-2-like protein that binds to and antagonizes the protective effect of Bcl-2, rendering cells more sensitive to apoptosis (27). In the present study, we found that only the level of Bax in $\mathrm{H} 28$ cells was significantly elevated by BBI treatment. As a result, the balance between pro- and anti-apoptotic factors was altered towards the induction of apoptosis. Any approach that alters the balance in favor of apoptosis may thus confer a therapeutic benefit. Overall, our present results suggest that $\mathrm{Cx} 43$ induced by BBI treatment influences the balance between pro- and anti-apoptotic factors in the direction of apoptosis, possibly contributing to the improved sensitivity of $\mathrm{H} 28$ cells to cisplatin. At present, the reason why BBI enhanced the level of Bax remains unclear. From the present data, we can only speculate that the inactivation of Src signaling caused by BBI-induced elevation of $\mathrm{Cx} 43$ was associated with the up-regulation of Bax. Since it is known that Bax is located downstream in the Src signal pathway, it appears that the inhibition of Src signaling indirectly induces Bax in $\mathrm{H} 28$ cells treated with BBI. In order to clarify the mechanism by which the enhancing effect of BBI on cisplatin-induced cytotoxicity in $\mathrm{H} 28$ cells is activated, further study is warranted.

\section{Acknowledgements}

This study was supported by a research grant for Health Sciences Focusing on Drug Innovation from the Japan Health Sciences Foundation (KHC1023), and a grant-in-aid for Science Research from the Ministry of Education, Culture and Sciences of Japan (no. 22500754) to Yano T.

\section{References}

1. Carbone M, Kratzke RA and Testa JR: The pathogenesis of mesothelioma. Semin Oncol 29: 2-17, 2002.

2. Nowak AK, Lake RA, Kindler HL and Robinson BW: New approaches for mesothelioma: biologics, vaccines, gene therapy, and other novel agents. Semin Oncol 29: 82-96, 2002.

3. Ryan CW, Herndon J and Vogelzang NJ: A review of chemotherapy trials for malignant mesothelioma. Chest 113: S66-S73, 1998.

4. Van Haarst JM, Baas P, Manegold CH, et al: Multicentre phase II study of gemcitabine and cisplatin in malignant pleural mesothelioma. Br J Cancer 86: 342-345, 2002.

5. Vinken M, Vanhaecke T, Papeleu P, et al: Connexins and their channels in cell growth and cell death. Cell Signal 18: 592-600, 2006.

6. Laird DW: Life cycle of connexins in health and disease. Biochem J 394: 527-543, 2006.

7. Mesni M, Krutovskikh V, Piccoli C, et al: Negative growth control of HeLa cells by connexin genes: connexin species specificity. Cancer Res 55: 629-639, 1995.
8. Sato $H$, Iwata $H$, Takano $Y$, Yamada $R$, Okuzawa $H$, Nagashima Y, Yamaura K, Ueno K and Yano T: Enhanced effect of connexin 43 on cisplatin-induced cytotoxicity in mesothelioma cells. J Pharm Sci 110: 466-475, 2009.

9. Kennedy AR: The Bowman-Birk inhibitor from soybeans as an anticarcinogenic agent. Am J Clin Nutri 68: S1406-S1412, 1998.

10. Armstrong WB, Kennedy AR, Wan XS, et al: Single-dose administration of Bowman-Birk inhibitor concentrate in patients with oral leukoplakia. Cancer Epid Biomark Prev 9: 43-47, 2000.

11. Billings PC, Newberne PM and Kennedy AR: Protease inhibitor suppression of colon and anal gland carcinogenesis induced by dimethylhydrazine. Carcinogenesis 11: 1083-1086, 1990.

12. Kennedy AR: Prevention of carcinogenesis by protease inhibitors. Cancer Res 54: S1999-S2005, 1994.

13. Suzuki K, Yano T, Sadzuka Y, Sugiyama T, Seki T and Asano R: Restoration of connexin 43 by Bowman-Birk protease inhibitor in M5076-bearing mice. Oncol Rep 13: 1247-1250, 2005.

14. Saito T, Sato H, Virgona N, Hagiwara H, Kashiwagi K, Suzuki K, Asano R and Yano T: Negative growth control of osteosarcoma cells by Bowman-Birk protease inhibitor from soybean; involvement of connexin 43. Cancer Lett 253: 249-257, 2007.

15. Nishimura M, Saito T, Yamasaki H and Kudo R. Suppression of gap junctional intercellular communication via 5' CpG island methylation in promoter region of E-cadherin gene in endometrial cancer cells. Carcinogenesis 24: 1615-1623, 2003.

16. Nam S, Smith DM and Dou QP: Ester bond-containing tea polyphenols potently inhibit proteasome activity in vitro and in vivo. J Biol Chem 276: 13322-13330, 2001.

17. Tang M, Asamoto M, Ogawa K, et al: Induction of apoptosis in the LNCaP human prostate carcinoma cell line and prostate adenocarcinomas of SV40T antigen transgenic rats by the Bowman-Birk inhibitor. Pathol Int 59: 790-796, 2009.

18. Sato H, Senba H, Virgona N, Fukumoto K, Ishida T, Hagiwara H, Negishi E, Ueno K, Yamasaki $\mathrm{H}$ and Yano T: Connexin 32 potentiates vinblastine-induced cytotoxicity in renal cell carcinoma cells. Mol Carcinog 46: 215-224, 2007.

19. Krutovskikh VA, Piccoli $\mathrm{C}$ and Yamasaki H: Gap junction intercellular communication propagates cell death in cancerous cells. Oncogene 21: 1989-1999, 2002.

20. Duflot-Dancer A, Mesnil M and Yamasaki H: Dominantnegative abrogation of connexin-mediated cell growth control by mutant connexin genes. Oncogene 15: 2151-2158, 1997.

21. Fujimoto E, Sato H, Shirai S, Nagashima Y, Fukumoto K, Hagiwara H, Negishi E, Ueno K, Omori Y, Yamasaki H, Hagiwara $\mathrm{K}$ and Yano T: Connexin32 as a tumor-suppressor gene in a metastatic renal cell carcinoma cell line. Oncogene 24: 3684-3690, 2005.

22. Benati D and Baldari CT: SRC family kinases as potential therapeutic targets for malignancies and immunological disorders. Curr Med Chem 15: 1154-1165, 2008.

23. Tsao AS, He D, Saigal B, et al: Inhibition of c-Src expression and activation in malignant pleural mesothelioma tissues leads to apoptosis, cell cycle arrest, and decreased migration and invasion. Mol Cancer Ther 6: 1962-1972, 2007.

24. Pahujaa M, Anikin M and Goldberg GS: Phosphorylation of connexin43 induced by Src: regulation of gap junctional communication between transformed cells. Exp Cell Res 313: 4083-4090, 2007.

25. Kim R, Emi M, Tanabe K, et al: Therapeutic potential of antisense Bcl-2 as a chemosensitizer for cancer therapy. Cancer 101: 2491-2502, 2004.

26. Chao DT and Korsmeyer SJ: BCL-2 family: regulators of cell death. Ann Rev Immunol 16: 395-419, 1998.

27. Hockenbery DM: Targeting mitochondria for cancer therapy. Environ Mol Mutagenesis 51: 476-489, 2010. 